\title{
Certain modifications to simplify arytenoid adduction technique for correction of paralytic dysphonia
}

\begin{abstract}
The Arytenoid Adduction (A.A) technique as originally described by Professor Isshiki of Japan, added a new dimension for improvement of voice in cases of paralytic dysphonia particularly in large posterior commissure defects. The present study is based on modifications done simplifying the standard technique of A.A described by Isshiki with required degree of medial rotation of vocal process and achievement of optimum voice in cases of paralytic dysphonia.
\end{abstract}

Keywords: arytenoid adduction technique, paralytic dysphonia, laryngeal frame work surgery, unilateral vocal fold paralysis, phonosurgery
Volume 10 Issue 4 - 2018

\author{
Phaniendra Kumar V,' Mohan Kumar R² \\ 'Director Sri Sathya Sai Institute of Otolayngology and Research \\ Centre for Voice Disorders, India \\ ${ }^{2}$ Speech Language Pathologist, Sri Sathya Sai Institute of \\ Ototolarngology, India
}

\begin{abstract}
Correspondence: $\mathrm{V}$ Phaniendrakumar, Professor Emeritus ENT, Director Sri Sathya Sai Institute of Otolaryngology and Research Centre for Voice Disorders, Guntur, AP, India, Tel +91 98481340 18, Email drvoicep@hotmail.com
\end{abstract}

Received: August 30, 2017| Published: July 17, 2018

\section{Abbreviations: ML, medialization laryngoplasty; AA, arytenoid adduction \\ Introduction \\ Phonosurgery for correction of paralytic dysphonia was started as early as 1915 by Payar who first reported medialization procedure through the anteriorly based cartilage flap.}

Selection of phonosurgical procedures in cases of paralytic dysphonia depends on the severity of patient's symptoms, glottic configuration, the tone of the paralyzed cord, and status of paralysis (Temporary or Permanent). Prof Isshiki, of Japan who first standardized the technique of medialization of vocal fold with silicone implant also had the credit of introducing for the first time the technique of Arytenoid Adduction (A.A) for correction of paralytic dysphonia. ${ }^{1,2}$ Arytenoid adduction (AA) is a framework surgery where the pull of the LCA muscle is imitated to achieve vocal fold repositioning. AA physiologically lowers the position of the vocal process, medializes and stabilizes the vocal process, lengthens the vocal fold, and rotates the arytenoid cartilage. Since arytenoid adduction (AA) is an irreversible procedure, it is adapted only in long standing, uncompensated, unilateral vocal cord paralysis with breathy dysphonia and also in cases where it is ascertained that the neuronal function of the affected vocal cord will not return to normal as shown by Laryngeal EMG. AA is also indicated in high vagal lesions with a triangular glottic defect of more than $2 \mathrm{~mm}$ and also in cases where the paralysed vocal cord is at a much lower level when compared to normal cord, as observed by fiberoptic laryngoscopy or stroboscopy. In these cases AA is done as a primary procedure along with medialization laryngoplasty or secondary procedure when the Medializatio Laryngoplasty (M.L) has failed to give sufficient voice improvement due to large posterior glottic gap. In the standard technique of A.A described by Isshiki achievement of optimum voice is excellent but the procedure is technically rather difficult and time consuming. As the prolene used to pull and rotate arytenoid was lengthy with different curves passing through the cricoid and laryngoplasty window difficult to rotate the arytenoid with a possibility of prolene cutting through.
According to Blake Simpson, Clark Rosen ${ }^{3}$ as only mild-moderate tension is required on the AA suture to achieve desirable arytenoid positioning, they described a technique to fix the prolene on the thyroid lamina through a hole made in the thyoiod cartilage at the intersection of two guidelines a vertical three forth distance from thyroid notch to inferior border and a horizontal line one third distance from midline of thyroid cartilage to inferior constrictor muscle border. But this point of fixation of prolene at the intersection these guide lines may not be possible in all the cases as the point of fixation mostly depends on the end point of traction on the prolene where the patient can produce optimum and unstrained voice. Our present study is based mostly on voice improved on the table rather than pleasing position of the vocal fold.

\section{Material \& methods}

45 patients, 30 males and 15 females under the age group of 18 to 67 years with paralytic dysphonia attending Sri Sathya Sai Institute of ENT and voice clinic at Guntur A.P India from January 2006 to-January 2016 were taken up for study. All these patients were subjected to the preliminary investigations for paralytic dysphonia including Stroboscopy, CT, Flexible fiber optic laryngoscopy with dynamic phonation, and audio recording of voice with perceptual voice analysis and palpation of arytenoids under DL scopy. Among the 45 cases that underwent A.A for correction of paralytic dysphonia, 35 cases had primary arytenoid adduction procedure combined with Medialization Laryngoplasty (M.L) by Sandwich thyroplasty technique and 10 cases A.A as secondary procedure supplementary to previously operated M.L cases

\section{Surgical technique}

Informed consent from all patients and approval from ethical committee were obtained all the patients were operated under local anaesthesia with optimum sedation under the supervision of anesthetist. Patient was kept in supine position with minimal extension of neck and little tilting of head to the normal side. With marker pen transverse incision of about 4 inches in length was marked from the 
midline at the midpoint of the thyroid lamina to the middle of the sternomastoid on the paralysed side. $1 \%$ Xylocaine with 1 in $1,00,000$ adrenaline was infiltrated in and around the incision area. After making the skin incision the superficial fascia and platysma were incised, the superficial jugular veins were ligated and the strap muscles were clamped, cut and coagulated. The thyroid lamina was exposed on all its sides. In cases of primary AA.Technique, the thyroplasty or M.L window was designed by Sandwich thyrolpasty technique and kept ready for medialization laryngoplasty. Inferior constrictor muscle fibers were divided at the posterior border of thyroid lamina with cautery. The posterior edge of the thyroid cartilage was lifted up with a hook and the cricothyroid joint identified and divided. $1 \mathrm{~cm}$. cephalically from the cricothyriod joint, the cricoarytenoid joint was identified and confirmed by palpating with the finger. Using a blunt tipped scissors cricoarytenoid joint was divided, exposing its glistening articular surface over the cricoid cartilage. At this stage one must be careful not to injure the bulging pyriform sinus mucosa. The muscular process of the arytenoids was freed of its muscular attachments. A 4 zero prolene with double-armed needle was passed through the muscular process of the arytenoid including its muscle remnants and passed anteriorly along the medial surface of the thyroid ala to imitate the pull of the LCA muscle. The inferior horn was released and brought to its original position. The prolene was pulled along the direction of action of lateral cricoarytenoid muscle, asking the patient to phonate for observing the improvement of voice.

The end point of the traction on the prolene and rotation of arytenoids totally depended on obtaining unstrained and optimum voice on the operating table. The end point was marked anterior inferiorly on the medial surface of the thyroid lamina. At this point the two ends of the prolene were brought out onto the lateral surface of thyroid lamina through 2 holes made in the lamina separately either directly piercing the lamina or with cautery if the lamina was very much calcified Adduction of the arytenoid achieved was also confirmed with the help of transnasal fiber optic video laryngoscope. The prolene was tied over silicone button on the lamina while observing the patient making optimum and unstrained voice (Figure 1) (Figure 2). Medialization laryngoplasty with silastic implant by Sandwich Thyroplasty4 technique was followed in all primary arytenoid adduction procedures after the completion of the Arytenoid Adduction procedureThe wound is closed in layers with a drain and dressed. The patient is kept on systemic antibiotics for about one week and steroids for about 4 days post - operatively.

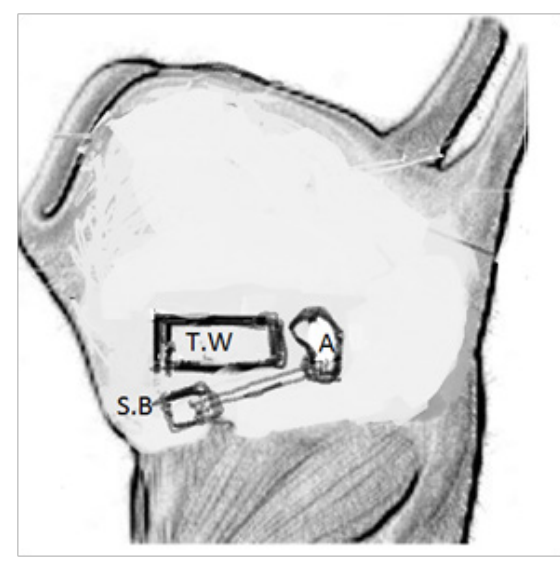

Figure I Arytenoid Adduction technique:- Prolene knot fixed over a Silastic Botton on the Anterioinferior part of lateral surface of the Thyroid Lamia A:Arytenod TW:- Thyroplasy Window S.B:- Silasitc Button.

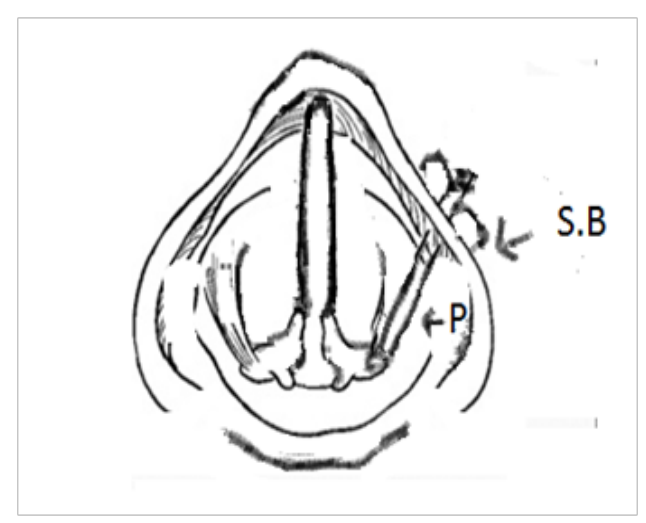

Figure 2 Arytenoid Adduction Technique:- Muscular Process of Arytenoid rotated along the direction Lateral Cricoarytenoid muscle and fixed in position as Prolene tied over the Silastic Button over the Thyroid Lamina P:Prolene S.B:- Silastic Button.

\section{Results}

The pre-operative and post-operative voice qualities of each patient were rated using Voice Rating scale by a speech pathologist.

\section{Normal \\ 2. Near normal (mild Dysphonia) \\ 3. Moderate dysphonia \\ 4. Severe Dysphonia \\ 5. Aphonia}

Of the30 patients who underwent primary AA procedure with medialization laryngoplasty (ML) 25 cases had voice score of 1 and 5 cases voice scores of 2 . Of the 15 patients who underwent AA as secondary procedure after preliminary ML with score of 3 had improvement of voice and attained score 1 . This improvement was due to closure of posterior commissure defect by AA procedure.

\section{Complications}

Review of literature reveals that the complication rate is higher in AA procedure compared to simple medialization laryngoplasty. The complications described are wound infection, slipping of arytenoids suture, pharyngocutaneous fistuala, carotid artery injury and airway obstruction. Among the 45 cases that underwent AA procedure only one case, a female aged 65years, had airway obstruction during third post-operative day due to retrolaryngeal hematoma necessitating a tracheostomy temporarily. In the all other cases the recovery was uneventful.

\section{Summary}

Arytenoid adduction (AA) is used in the treatment of glottal insufficiency. Unlike medialization laryngoplasty, AA acts through direct traction on the arytenoid cartilage at the muscular process, mimicking the action of the lateral cricoarytenoid muscle. AA is an important adjunct in selected cases of vocal fold paralysis like in patients with vocal fold paralysis who have a lack of vocal process contact during phonation (large posterior gap), shortened immobile vocal fold, and those with vocal folds at different levels, Since its introduction by Prof. Isshiki, Arytenoid adduction technique has been widely practiced by phonosurgeons in many parts of the world. The 
results of combined M.L. and A.A. procedure in respect of voice improvement were much superior to results produced by Medialisation Laryngoplastry (M.L) alone in properly indicated cases. A has been proved to be a very useful procedure in preventing aspirations due to severe glottic incompetence as in high vagal lesions. Since it is an irreversible and complicated procedure compared to Medialization (M.L) one must be sure that the neuronal function of the paralysed cord will not return to normal. AA has limited application in patients with previous external beam radiation to neck, in case of radical neck surgery, in diabetics or in patients who are immune-suppressed from cancer chemotherapy. The present study is a modification for simplifying standard technique of Arytenoid Adduction for correction of paralytic dysphonia. It is almost similar to technique described by Black Simpson and Clark Rosen but differs from it on the principle of fixation of prolene on thyroid lamina based on voice improvement rather than anatomical guidelines.

\section{Conflict of interest}

The author declares there is no conflict of interest.

\section{References}

1. Isshiki N, Mortin H, Okamura H, et al. Thyroplasty as a new phonosurgical technique. Acta Otolarngol. 1974;78(5-6):451-457.

2. Isshiki N, Tanable M, Swada M. Arytenoid Addution for Unilateral Vocal Cord Paralysis. Arch Otolaryngol. 1978;140:555-558.

3. Blake Simpson, Clark Rosen. Operative techniques in Otolaryngology.

4. Phaniendrakumar V, Chaitanya CB, Ravindranath TA, et al. Sandwich Thyroplasty: A Novel Technique for Simplifying Medialization of Vocal Fold Using Silicone Implant in Paralytic Dysphonia with Modification of Thyroplasty Window. Indian J Otolaryngol Head Neck Surg 2015;67(2):159-164.

\section{Acknowledgments}

None. 